\title{
Perspectiva de aplicação do método Analytic Hierarchy Process no cenário brasileiro de pesquisa
}

\section{Analytic Hierarchy method perspective in the Brazilian research scenario}

\author{
FLAVIA CRISTINA DA SILVA \\ Universidade Presbiteriana Mackenzie \\ FABIO ITOSHI SHIBAO \\ Universidade Anhembi-Morumbi \\ ANDRÉ FELIPE HENRIQUES LIBRANTZ \\ Universidade Nove de Julho \\ MÁRIO ROBERTO DOS SANTOS \\ Universidade Nove de Julho \\ GERALDO CARDOSO OLIVEIRA NETO \\ Universidade Nove de Julho
}

\section{RESUMO}

Este estudo teve por objetivo mapear o emprego do método Analytic Hierarchy Process (AHP) como estratégia ou objeto de pesquisa nos programas brasileiros de pós-graduação stricto sensu indexados no acervo da Biblioteca Digital Brasileira de Teses e Dissertações até o ano de 2016, além de estabelecer comparações e apontar as principais tendências. Os procedimentos técnicos consistiram em pesquisa bibliográfica para a coleta e técnicas de estatística descritiva e análise de redes sociais para tratamento e análise dos dados. Foram identificados 383 documentos, o que significa que o método AHP foi empregado em, aproximadamente, $0,734 \%$ do total de pesquisas. As áreas de Engenharias e Ciências Sociais Aplicadas concentraram mais de $85 \%$ dos estudos, que, em sua maioria, estiveram voltados aos problemas de decisão no setor de infraestrutura, que abrange as atividades de planejamento territorial e urbano, construção civil, energia, telecomunicações e transportes. Constatou-se que 
o método AHP se encontra em estágio embrionário no cenário brasileiro de pesquisa, entretanto a linha de tendência sugere pronunciado aumento nos próximos anos. Este estudo traz contribuição metodológica na medida em que detalha, exemplifica e orienta o uso do método AHP, ao mesmo tempo em que colabora para as decisões gerenciais ao identificar os problemas de decisão e os setores econômicos mais aderentes à pesquisa.

Palavras-chave: Analytic hierarchy process (AHP). Análise de redes sociais (ARS). Decisão multicritério. Revisão sistemática.

\section{Abstract}

The purpose of this study was to map the use of the Analytic Hierarchy Process (AHP) method as a strategy or object of research in the Brazilian post-graduate programs stricto sensu indexed in the Brazilian Digital Library of Theses and Dissertations until the year 2016. establish comparisons and point out the main trends. The technical procedures consisted of bibliographic research for the collection and techniques of descriptive statistics and analysis of social networks for data treatment and analysis. A total of 383 documents were identified, which means that the AHP method was used in approximately $0.734 \%$ of the total research. The areas of Engineering and Applied Social Sciences concentrated more than $85 \%$ of the studies, which, for the most part, were focused on decision problems in the infrastructure sector, which encompasses territorial and urban planning, civil construction, energy, telecommunications and transport. It was found that the AHP method is at an embryonic stage in the Brazilian research scenario, however the trend line suggests a sharp increase in the coming years. This study brings a methodological contribution to the extent that it details, exemplifies and guides the use of the AHP method, at the same time that it collaborates for managerial decisions in identifying the decision problems and the economic sectors most adherent to the research.

Keywords: Analytic hierarchy process (AHP). Multicriteria decision. Systematic review. Social networking analysis. 


\section{INTRODUÇão}

Um processo de tomada de decisão consiste em selecionar e implementar a solução mais apropriada para alcançar o melhor nível de desempenho. Espera-se que essa tomada de decisão seja conduzida de maneira confiável, buscando maximizar os efeitos derivados de fatores positivos, e, simultaneamente, minimizar os fatores negativos (BENÍTEZ et al., 2019).

A maioria das decisões tomada baseia-se na intuição e no bom senso. As decisões mais complexas exigem uma abordagem mais sistemática e adoção de metodologias apropriadas de apoio à decisão. Os problemas de decisão complexos exigem considerar uma pluralidade de pontos de vista, tecnicamente chamados de critérios, conceitos e metodologias de múltiplos critérios (ABASTANTE et al., 2019).

Entre as técnicas que apoiam o processo de tomada de decisão, o Analytic Hierarchy Process (AHP) é uma das mais frequentemente utilizadas (B ALUSA; GORAI, 2019; KHATWANI; KAR, 2017; MATOS et al., 2018). É uma técnica de análise multicritério (multi-criteria decision-making - MCDM) que utiliza comparações pareadas, baseadas em uma escala numérica, sistematizando e estruturando o processo (SANTOS et al., 2019).

Este estudo teve por objetivo mapear o emprego do método Analytic Hierarchy Process (AHP) como estratégia ou objeto de pesquisa nos programas brasileiros de pós-graduação stricto sensu, além de estabelecer comparações e apontar tendências no uso para as áreas de Administração e Engenharia.

O desenvolvimento de uma perspectiva abrangente sobre as atividades de pesquisa associadas às aplicações desse método fornece insights sobre as contribuições de países, institutos, autores e periódicos para os avanços do método (ZYOUD; FUCHS-HANUS$\mathrm{CH}, 2017)$.

Após esta breve introdução, seguem-se outras cinco seções: o referencial teórico é apresentado na segunda seção, detalha-se o método de pesquisa na seção três, os resultados são apresentados na seção quatro e discutidos na quinta seção e, por fim, a seção seis encerra o estudo com as considerações finais, contribuições para a teoria e prática e sugestões para futuros estudos. 


\section{REFERENCIAL TEÓRICO}

Neste referencial, serão apresentadas a Teoria da decisão e a Escola Americana; o método de análise hierárquica AHP; o rigor matemático do método; as ferramentas eletrônicas para aplicação; críticas ao método AHP; e variações do método AHP.

\subsection{Teoria da Decisão e a Escola Americana}

A tomada de decisão é um problema enfrentado comumente pelo ser humano, tanto em tarefas simples quanto complexas, que pode ser orientada por um único ou múltiplos parâmetros de escolha e consiste na coleta de informações, busca de potenciais alternativas e seleção dessas alternativas (GOMES; GOMES; ALMEIDA, 2009).

Grande parte dos interesses práticos requer a análise global de vários atributos, em que um conjunto de alternativas está associado a um conjunto de critérios ou atributos e a um conjunto de consequências (PARREIRAS, 2006). A análise multicritério tem por finalidade assistir as escolhas dos seres humanos em relação à diversidade de elementos envolvidos em um processo decisório, inclusive incerteza, conveniência e antagonismos, entre outros (BOUYSSOU,1990).

A Escola Americana é tida como a precursora na análise multicritério devido à teoria da utilidade, inicialmente proposta em 1738 por Bernoulli e formalizada por meio de fundamentação axiomática somente em 1944 por Neumann e Morgenstern (PARREIRAS, 2006). A Escola Americana se caracteriza como normativa ou prescritiva, uma vez que o decisor utiliza sua experiência prévia para optar entre os modelos propostos sem alterá-los (LIEGGIO JR; GRANEMANN; SOUZA, 2012).

Os métodos da Escola Americana são também conhecidos como compensatórios, visto possuírem a função de agregação ou síntese das informações, por meio da qual o baixo desempenho de uma alternativa em um dado critério pode ser compensado pelo alto desempenho desta mesma alternativa em outro critério (GOMES; GOMES; ALMEIDA, 2009).

Os principais métodos são AHP, MACBETH, UTA, SMARTS, SMARTS INTERVALAR, SMARTER, Programação por metas e UTADIS. Segundo Salomon (2010), destacam-se como mais utiliza- 
dos o Analytic Hyrarchy Process (AHP), Multi-Attribute Utility Theory (MAUT) e Measuring Attractiveness by a Categorical Based Evaluation Technique (MACBETH).

\subsection{Analytic Hierarchy Process (AHP)}

$\mathrm{O}$ método de análise hierárquica AHP pode ser compreendido como uma teoria geral de medição que tem por finalidade apoiar o processo de tomada de decisão, com base no pensamento dedutivo e indutivo. É elegível tanto para critérios quantificáveis quanto intangíveis que se prestem desde o planejamento à resolução de conflitos, tendo por orientação o conhecimento e experiência adquiridos (SAATY, 1987; VARGAS, 1990). Foi proposto para construir a priorização de cada alternativa com base nas comparações pareadas de cada objeto com todas as outras (AB ASTANTE et al., 2019).

A essência do método consiste na decomposição, julgamentos comparativos e estabelecimento de prioridades como detalhado na Figura 1.

O AHP é aplicável aos dois tipos de comparações realizadas pelos seres humanos: comparação absoluta, na qual as alternativas são comparadas a um padrão, seja ele existente na memória ou desenvolvido por meio de experiência, e a comparação relativa, quando as alternativas são comparadas aos pares de acordo com um atributo comum (SAATY; VARGAS, 2012a).

\section{ETAPAS 1 A 5}

Descrevem a estruturação do problema com seus objetivos, atributos (critérios e subcritéiros) e alternativas, em forma de hierarquia, observando-se a homogeneidade dos elementos alocados em cada nível (SAATY, 1986).

Uma estrutura hierárquica é a forma mais simples de exibir a dependência funcional entre níveis componentes de um sistema, e é também um modo conveniente de decompor um problema complexo, pois retrata o relacionamento entre os elementos agrupados em níveis, com vistas às explicações de causa e efeito nas etapas que formam a cadeia (SAATY,1986,1994), como mostra a Figura 2. 
Figura 1 - Passos para aplicação do método AHP

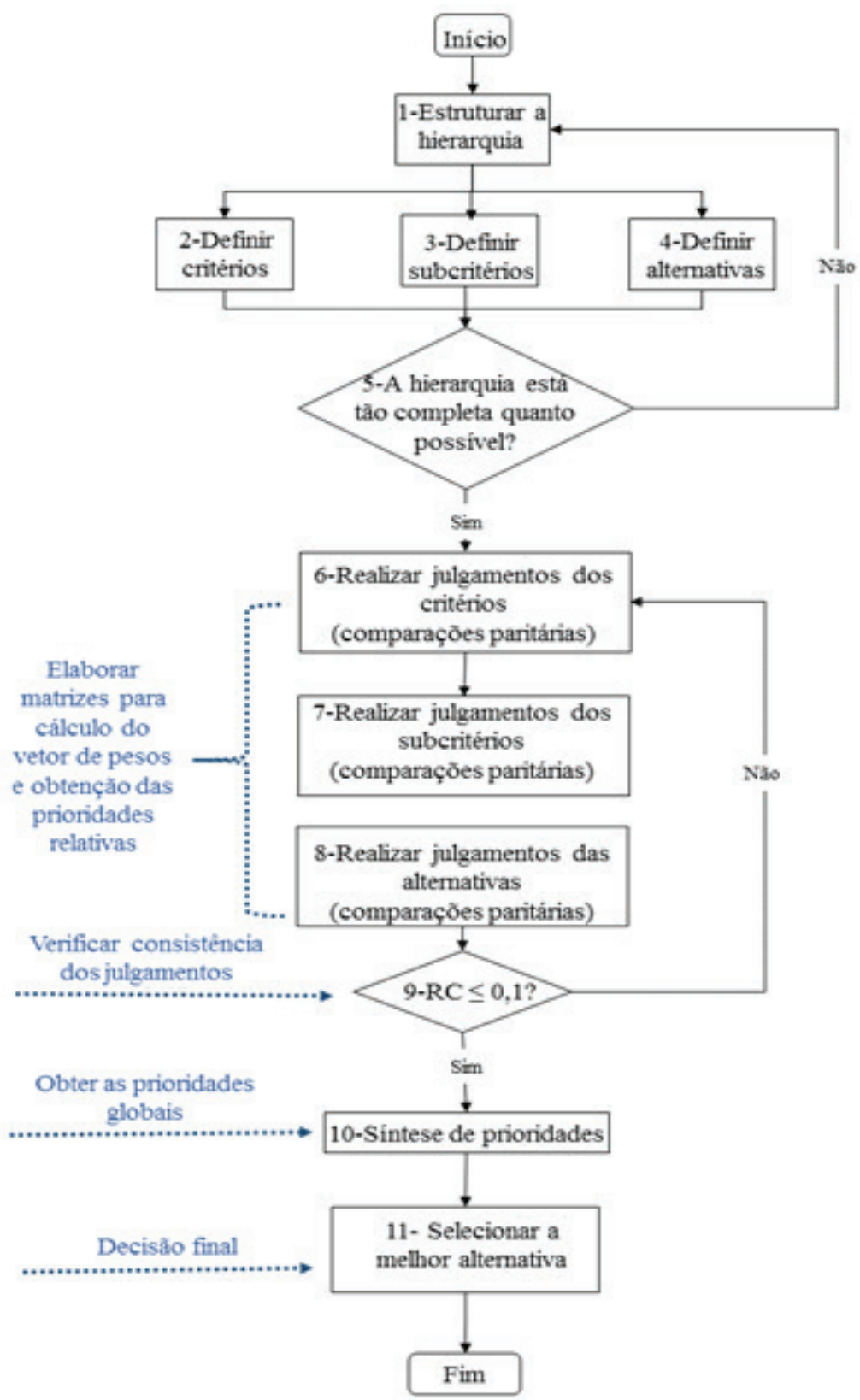

Fonte: Elaborado pelos autores 
Figura 2 - Estrutura hierárquica do método AHP

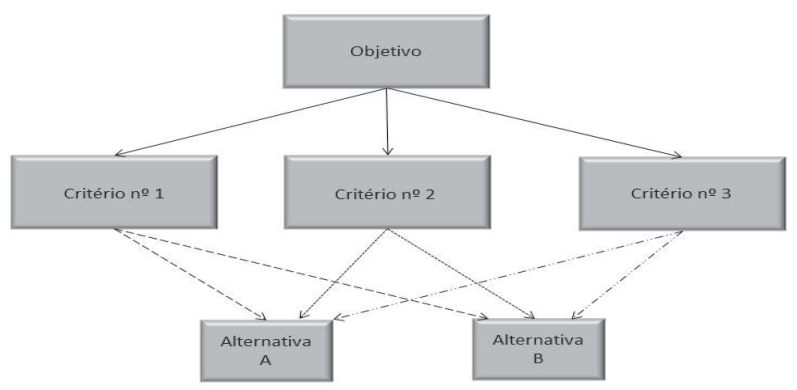

Fonte: Saaty (1977)

A formação dispõe de plasticidade suficiente para a criação de um nível intermediário entre critérios e alternativas e a inclusão de subcritérios (etapa 3), caso o problema requeira essa necessidade, pois, conforme ressaltado por Saaty $(1987 ; 1990)$, o cenário para a construção das hierarquias deve ser o mais detalhado quanto possível, contudo não a ponto de descaracterizar os elementos que o compõem (etapa 5). A validação da estrutura hierárquica, ou seja, a comprovação de sua consistência, pode ser verificada se os elementos do nível superior servirem como atributos comuns para os elementos do nível imediatamente inferior (SAATY, 1994).

\section{ETAPAS 6 A 8}

As prioridades de um critério ou subcritério sobre outro, ou de uma alternativa sobre outra, são estabelecidas por meio de comparações, par a par, em razão de uma única propriedade por vez, de modo que o decisor, fundamentado na observação ou experiência, determina a importância relativa entre eles, sem preocupação com a influência de outras propriedades ou importância de outros elementos (SAATY, 1990).

A escala fundamental do método AHP representada na Figura 3, permite a derivação de números absolutos em razão de priori- 
dades, e consiste em uma ferramenta de informação e comunicação de significados que utiliza palavras para representar os conceitos envolvidos nas decisões, dessa forma, é oferecido um equivalente semântico, chamado de escala verbal a cada índice de importância (SAATY, 2006).

Figura 3 - Escala fundamental

\begin{tabular}{|l|l|l|}
\hline $\begin{array}{l}\text { Escala } \\
\text { numérica }\end{array}$ & $\begin{array}{l}\text { Escala } \\
\text { Verbal }\end{array}$ & Definição \\
\hline 1 & $\begin{array}{l}\text { Igualmente } \\
\text { importante }\end{array}$ & $\begin{array}{l}\text { Duas alternativas contribuem igualmen- } \\
\text { te para o objetivo. }\end{array}$ \\
\hline 3 & $\begin{array}{l}\text { Moderadamente } \\
\text { importante }\end{array}$ & $\begin{array}{l}\text { Entre duas alternativas, por experiência } \\
\text { e julgamento, uma se apresenta discreta- } \\
\text { mente mais importante que a outra. }\end{array}$ \\
\hline 5 & $\begin{array}{l}\text { Significativa- } \\
\text { mente impor- } \\
\text { tante }\end{array}$ & $\begin{array}{l}\text { Entre duas alternativas, por experiência } \\
\text { e julgamento, uma se apresenta significa- } \\
\text { tivamente mais importante que a outra. }\end{array}$ \\
\hline 7 & $\begin{array}{l}\text { Muito fortemen- } \\
\text { te importante }\end{array}$ & $\begin{array}{l}\text { Entre duas alternativas, a importância } \\
\text { de uma é demonstrada mais expressiva- } \\
\text { mente que a outra. A relação de domi- } \\
\text { nância pode ser demonstrada na prática. }\end{array}$ \\
\hline 9 & $\begin{array}{l}\text { Extremamente } \\
\text { importante }\end{array}$ & $\begin{array}{l}\text { ta absolutamente mais importante que a } \\
\text { outra. Evidências conferem o mais alto } \\
\text { grau de certeza. }\end{array}$ \\
\hline $2,4,6^{*}$ & $\begin{array}{l}\text { Valores inter- } \\
\text { mediários entre } \\
\text { dois julgamentos }\end{array}$ & $\begin{array}{l}\text { Quando é necessário ponderar a atribui- } \\
\text { ção entre dois valores }\end{array}$ \\
\hline
\end{tabular}

*Os valores 2,4 e 6 são considerados intermediários e devem ser utilizados em caso de dúvida, quando há necessidade de ponderar a atribuição entre dois valores da escala.

Fonte: Saaty (2006)

Uma vez realizados os julgamentos pelos decisores, o cálculo das prioridades relativas é realizado por meio de matrizes de cálculo em planilhas eletrônicas e outras ferramentas automatizadas. De acordo com Trevisano (2007), normalmente há um software correspondente à aplicação de cada método multicritério, com o intuito 
de que o decisor esteja desobrigado de que cálculos complexos se concentrem na análise do problema e busca da solução. Por outro lado, alguns autores, tais como Millet e Saaty (2000), advertiram que os softwares aplicativos do método AHP tendem a omitir informações importantes do decisor, uma vez que normalizam os resultados antes de apresentá-los.

\section{ETAPA 9}

De acordo com Millet e Saaty (2000), as pessoas possuem habilidade de estabelecer relações coerentes entre elementos diversos, porém, não obstante as decisões estejam apoiadas em conhecimento e experiência, podem apresentar inconsistências, principalmente frente a um número elevado de comparações paritárias.

Saaty e Hu (1998) defenderam que essa taxa mínima de inconsistência corresponde aos novos conhecimentos que, uma vez agregados à experiência do decisor, tendem a alterar a ordem de preferência das alternativas e favorecer a criação de novos modelos de solução.

O Consistency Random (CR) é a proporção de consistência da matriz, quando comparada a um índice randômico (random índex IR), conforme a Tabela 1.

\section{Tabela 1 - Índice randômico}

\begin{tabular}{l|l|l|l|l|l|l|l|l|l|l}
\hline $\mathrm{n}$ \\
$\begin{array}{l}\text { (Número de } \\
\text { ordem da } \\
\text { matriz) }\end{array}$ & 1 & $\mathbf{2}$ & $\mathbf{3}$ & $\mathbf{4}$ & $\mathbf{5}$ & $\mathbf{6}$ & $\mathbf{7}$ & $\mathbf{8}$ & $\mathbf{9}$ & $\mathbf{1 0}$ \\
\hline $\mathrm{IR}$ & 0 & 0 & 0,58 & 0,90 & $\mathbf{1 , 1 2}$ & 1,24 & 1,32 & 1,41 & 1,45 & 1,49 \\
\hline
\end{tabular}

O número de ordem equivale ao número de elementos (critérios, subcritérios ou alternativas) da matriz de julgamento de prioridades. Nota-se que o cálculo de CR é desnecessário para matrizes de até dois elementos.

Fonte: Saaty (2014)

Embora seja admissível que o percentual de inconsistência de uma matriz possa alcançar até 20\% (FUNO; MUNIZ JR; MARINS, 2013; UNIKASARI et al., 2013) recomenda-se que não exceda a $10 \%$, $(\mathrm{CR} \leq 0,1)$, a fim de que as perturbações sejam pequenas e não 
venham a comprometer a grandeza das respostas, assim, o número de alternativas a serem comparadas paritariamente, ou o número de ordem da matriz, estariam também limitados a, no máximo, dez (SAATY; VARGAS, 2012a, b). Todavia, caso o valor do CR não atenda aos limites pré-determinados, Saaty e Vargas (2012a, b) recomendaram que o decisor reavalie seus julgamentos.

\section{ETAPA 10}

Qualquer que seja a ferramenta, no método AHP, as prioridades, em geral, são obtidas pela normalização do vetor de prioridades, ou seja, pelo cálculo do autovetor direito (SAATY; HU, 1998; SAATY, 1991), não obstante diversos métodos tenham sido propostos em substituição, tais como o cálculo da média das colunas normalizadas, a média das linhas normalizadas, a média geométrica das linhas, entre outras (GOMES; GOMES; ALMEID A, 2009).

A normalização se refere ao conceito de escassez e abundância quanto à presença dos critérios em cada alternativa, de modo que esse contraste determina a classificação de prioridade. A distribuição de um critério abundante exerce pouca ou nenhuma influência na determinação da importância das alternativas, ao passo que um critério escasso é mais influente no grau de dominância entre as alternativas, porque mais dele estará presente na alternativa dominante (SAATY, 2006).

\section{ETAPA 11}

Após os passos anteriores, as alternativas se diferenciam por valores, de modo que o maior valor aponta a alternativa com maior prioridade.

\subsection{Rigor Matemático do Método}

O método AHP se utiliza dos princípios da álgebra linear para a modelagem de questões empíricas, a partir da inferência de que não apenas os problemas, como o conhecimento, métodos utilizados e também os objetivos pretendidos são relativos (SA ATY, 1994). Com base na escala de medição da composição hierárquica, quatro fundamentos axiomáticos embasam o método: 
Axioma 1 - Reciprocidade: os elementos comparados aos pares pelo decisor devem ser reciprocamente correspondentes dentro da matriz, ou seja, se o elemento $A$ é $x$ vezes mais preferido que $B, \log$ o o elemento $B$ será $x^{-1}$ vezes menos preferido que $A$ (SAATY, 1987). O AHP emprega uma matriz dita positiva e recíproca em que todos os elementos são positivos, e aqueles posicionados à esquerda da diagonal principal, são inversamente proporcionais quando comparados aos elementos da direita, como representado em (1).

$$
a_{i j}=\frac{1}{a_{j i}} \text { ou } a_{i j} a_{j i}=1, a_{i j}>0
$$

II) Axioma 2 - Homogeneidade: somente os elementos que pertençam a um mesmo conjunto ou que possuam características similares são comparáveis, uma vez que os erros de julgamento estão proporcionalmente associados ao grau de disparidade entre os elementos (SAATY, 1986). Dentro de um determinado nível, os elementos que ali se encontram devem possuir o mesmo grau de importância (SAATY, 1986). A condição de similaridade deve se restringir a um conjunto de alternativas em função de um determinado critério, de modo que as preferências sejam mostradas por meio de uma escala limitada (SAATY, 2006), como se exemplifica em (2).

$$
x \in L_{k}, x^{-} \subseteq L_{k+1}
$$

III) Axioma 3 - Independência: quando comparados paritariamente os elementos de um determinado nível da hierarquia devem ser mutuamente excludentes entre si, além do que o peso de cada critério deve ser atribuído independentemente das alternativas (SAATY, 1986), assim, em termos matemáticos, uma hierarquia $H$ contém os níveis $L_{1}, L_{2}, \ldots, L_{h}, \mathrm{Lh}$, para cada $L_{k}$, em que $k=1,2, \ldots, h-1$, equivale a dizer que:

a) $L_{k+1}$ é externamente dependente de $L_{k}$;

b) $L_{k+1}$ é internamente independente com relação a todos os elementos $a \in L_{k}$;

c) $L_{k}$ não é externamente dependente de $L_{k+1}$.

IV) Axioma 4 - Expectativas: para fins de tomada de decisão, a hierarquia é admitida como completa (SAATY, 2006). As crenças de cada pessoa são baseadas em razões individuais, de modo que, para garantir que o resultado retrate suas ideias, é necessário que todas as alternativas, critérios e expectativas, explícitas e implícitas, estejam representadas na hierarquia (SAATY, 1990). De acordo com Saaty (1986), o processo de análise hierárquica não se vincula estritamente à racionalidade, visto que as pessoas possuem muitas expectativas irracionais, estas podem ser acomodadas no processo decisório a partir de uma função de ponderação fundamentada nos conceitos do Axioma 3. Dito de outra forma, os critérios $C$ estão contidos na hierarquia $H$ composta pelos níveis $L_{h}$, que, por sua vez, são formados pelos elementos a (SAATY, 1986), como se mostra em (3).

$$
C \subset H-L_{h}, \quad a=L_{h}
$$




\subsection{Ferramentas eletrônicas e críticas ao método AHP}

O método AHP é amplamente documentado na literatura científica tanto em aplicações práticas na tomada de decisão quanto por correntes que realçam suas vantagens e desvantagens (SALOMON, 2010; VAIDYA; KUMAR, 2006).

Ossadnik e Lange (1999) relacionaram o potencial de difusão do método AHP ao uso de uma ferramenta computacional eficiente e descomplicada. De acordo com Trevisano (2007), normalmente há um software correspondente à aplicação de cada método multicritério, com o intuito de que o decisor esteja desobrigado de cálculos complexos e venha a concentrar maior atenção na análise do problema e busca da solução.

Há uma grande diversidade de softwares disponíveis para a aplicação do método AHP, tanto gratuitos quanto pagos, no entanto a maior parte desses aplicativos são compatíveis apenas com o modelo tradicional do método, em prejuízo para que se propaguem os métodos derivados como F-AHP e aplicações híbridas tais como as associações de AHP e DEA, QFD, análise SWOT, algorítimos genéticos, redes neurais e outros (ISHIZAKA; LABIB, 2009).

As avaliações positivas do AHP estão relacionadas à facilidade de aplicação (SAATY, 1990; SALOMON, 2010; SALOMON; MONTEVECHI, 2001), robustez alcançada com a estruturação formal do problema (GOMES; GOMES; ALMEID A, 2009; SA ATY, 1994) e fundamentação axiomática (WHITAKER, 2007), bem como possibilidade de verificação da consistência (PARREIRAS, 2006; SAATY, 2006).

Além de ser aplicável à análise de dados qualitativos e quantitativos (STEIGUER; DUBERSTEIN; LOPES, 2003), o AHP apresenta versatilidade e compatibilidade com outras metodologias (BARIN et al., 2010; LIMA et al. 2014; SOLTANI; MARANDI; IVAKI, 2013).

Por outro lado, os estudos que desaprovam o método ressaltam argumentos como a suposição em que se baseia a correspondência entre as escalas verbal e numérica (BELTON; GOODWIN, 1996), a indução ao erro de quantificação devido a utilização do autovalor para cálculo do vetor de prioridades, imprecisão do coeficiente de consistência em relação aos erros de quantificação na derivação dos vetores de prioridades (BANA e COSTA; VANSNICK, 2008). 
Outras fragilidades apontadas consistem na possibilidade de que, devido a uma interpretação equivocada, o decisor atribua pesos incorretos (LOOTSMA, 1990) e tendência de que os valores extremos (1 e 9) sejam excluídos dos julgamentos (BELTON; STEWART, 2002). Entretanto, maior ênfase é dada ao ranking reverso das alternativas, ou seja, mediante a exclusão da alternativa prioritária, a segunda colocada não necessariamente será tomada como a de maior prioridade (BARZILAI; GOLANI, 1994; BELTON; GEAR, 1985; LOOSTMA, 1990).

\subsection{Variações do Método AHP}

Ao longo do tempo, as discussões em torno do método AHP originaram variações focadas na melhoria dos principais pontos criticados pela comunidade científica. A seguir estão relacionadas as adaptações do método AHP mais difundidas na literatura (B ARIN et al., 2010; GOMES; GOMES; ALMEID A, 2009):

a) M-AHP ou Modified Analytic Hierarchic Process, proposto por Lootsma (1990) também conhecido como AHP multiplicativo, adotou a escala natural de -8 a +8 e agregação geométrica das preferências;

b) AHP Referenciado, proposto por Watson e Freeling (1982), inseriu uma constante $\mathrm{K}$ de proporcionalidade que cria uma restrição a ser considerada no cálculo da relevância dos critérios;

c) AHP B-G, proposto por Belton e Gear (1985), realiza a normalização das preferências a partir do maior valor, justamente o que é alcançado pela alternativa escolhida após a comparação binária;

d) F-AHP ou Fuzzy Analytic Hierarchic Process, conforme Naghadehi, Mikaeil e Ataei (2009), muitos autores propuseram formas variadas de aplicação do método F-AHP: Van Laarhoven e Pedrcyz (1983) sugeriram a aplicação da lógica fuzzy aos princípios do AHP; Buckley (1985) inseriu números fuzzy trapezoidais na avaliação das alternativas em complemento à proposição de Van Laarhoven e Pedrcyz (1983); Chang (1996) introduziu números fuzzy triangulares na escala de comparações pareadas; Deng (1999) apresentou a abordagem fuzzy como alternativa para contornar os frequentes problemas relacionados às decisões multicritérios, entre outros. 


\section{Procedimentos metodológicos}

Em relação à sua natureza, esta pesquisa classifica-se como básica e do ponto de vista de seus objetivos exibe contornos exploratórios. Tal como ressaltado por Prodanov e Freitas (2013) e Vieira (2007), tem a finalidade de disponibilizar mais informações sobre o assunto, no caso o emprego do método AHP como estratégia ou objeto de pesquisa nos programas brasileiros de pós-graduação stricto sensu.

A respeito do modus operandi, os procedimentos técnicos consistiram em pesquisa bibliográfica para a coleta e técnicas de estatística descritiva e análise de redes sociais (ARS) para o tratamento e análise dos dados. O universo da pesquisa foi limitado ao acervo da Biblioteca Digital Brasileira de Teses e Dissertações (BDTD), que reúne teses e dissertações defendidas em 85 instituições brasileiras e também por brasileiros no exterior. A amostra foi limitada aos trabalhos defendidos até o ano de 2016.

A busca foi sistematizada de acordo com os termos "análise hierárquica", "análise hierárquica do processo", "analytic hierarchy process" e "AHP" aplicados aos campos título, assunto, resumo Português e resumo Inglês. Os resultados considerados válidos abrangeram estudos em que o método AHP foi utilizado como estratégia de pesquisas, mesmo que associado a outros métodos, ou como tema ou objeto de pesquisas conceituais.

No fichamento dos documentos foram extraídos dados correspondentes ao tipo de documento, ano de defesa, instituição e programa, tema, tipo do método AHP e ferramenta de aplicação, setor econômico relacionado ao estudo, título, autor, orientador, co-orientador e membros da banca.

As métricas de ARS utilizadas foram grau de centralidade (GC) ou centrality degree, grau de intermediação (GI) ou betweeness centrality e classificação ou pagerank, com as quais se objetivou respectivamente: (i) avaliar o número de ligações de um elemento, tanto as que são orientadas para ele (indegree) como as que ele encaminha para outros nós (outdegree); (ii) verificar o número de vezes que um vértice age como ponte entre os demais; e (iii) identificar a importância do elemento (FREEMAN, 1996; WASSERMANN; FAUST, 1994). Para tanto, foi empregada a ferramenta NodeXL, uma extensão o software Microsoft Excel de código aberto e gratuita em sua versão 
básica, acessível no endereço eletrônico https://nodexl.codeplex.com/ (HANSEN; SHNEIDERMAN; SMITH, 2010).

\section{ApresentaÇão dos Resultados}

Os resultados primários apresentados na Tabela 2 incluem sobreposições dos filtros e trabalhos não alinhados ao objetivo deste estudo.

Tabela 2 - Dados brutos resultados da busca sistematizada

\begin{tabular}{|c|c|c|c|c|}
\hline \multirow[b]{2}{*}{ Termo de busca } & \multicolumn{4}{|c|}{ Campos } \\
\hline & Título & Assunto & $\begin{array}{l}\text { Resumo em } \\
\text { Português }\end{array}$ & $\begin{array}{l}\text { Resumo em } \\
\text { Inglês }\end{array}$ \\
\hline Análise hierárquica & 25 & 20 & 223 & 223 \\
\hline $\begin{array}{l}\text { Análise hierárquica } \\
\text { do processo }\end{array}$ & 5 & 4 & 31 & 31 \\
\hline $\begin{array}{l}\text { Analytic Hierarchy } \\
\text { Process }\end{array}$ & 18 & 40 & 284 & 284 \\
\hline AHP & 73 & 104 & 465 & 465 \\
\hline
\end{tabular}

Fonte: Dados da pesquisa

Após a depuração dos dados, por meio da exclusão de entradas duplicadas e trabalhos dissonantes dos critérios de seleção, obteve-se 383 resultados válidos, divididos em 312 dissertações e 71 teses, o que representa $0,0734 \%$ do total de 523.137 documentos indexados na base.

A Figura 4 ilustra a distribuição da produção científica no período de 1992 a 2016. Em relação às dissertações, a observação dos resultados condensados a cada cinco anos constatou a pronunciada tendência de maior emprego do método AHP: de 1998 a 2004 o método AHP foi empregado quase quatro vezes mais que nos primeiros cinco anos; de 2005 a 2009 o AHP foi utilizado 92\% mais vezes nas pesquisas de mestrado do que nos dez anos anteriores, enquanto em 2010 a 2014 os resultados alcançaram o dobro do período anterior. Os anos de 2015 e 2016, respectivamente com 42 e 43 dissertações, apresentaram quase três vezes a média de produção de todo o período. 
Figura 4 - Aplicação do método AHP

em pesquisas de pós-graduação stricto sensu

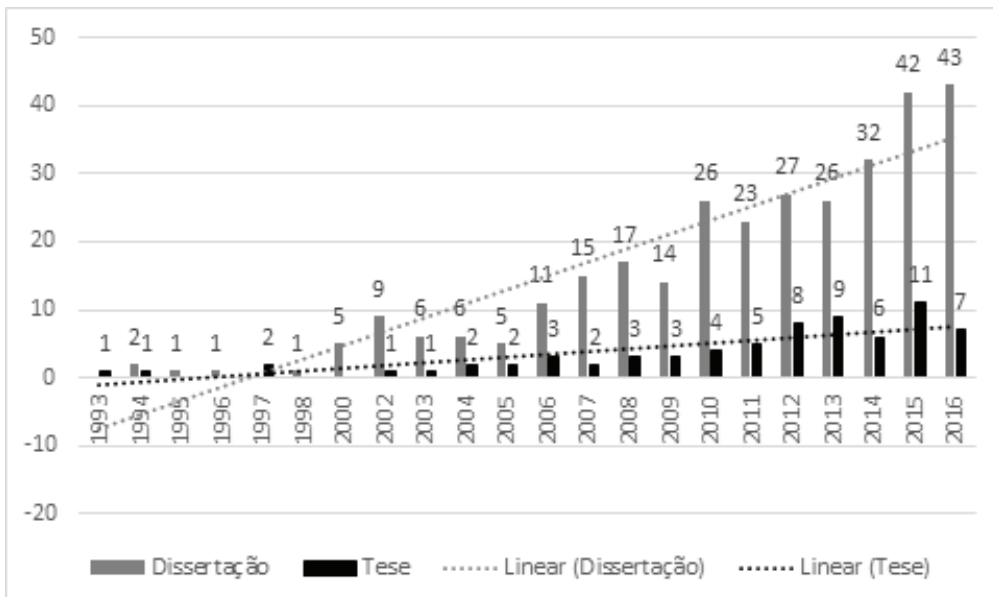

Fonte: Elaborada pelos autores a partir dos dados coletados

Embora a linha de tendência seja menos incisiva para teses, percebe-se que o número de pesquisas dos últimos cinco anos excedeu em mais de 30\% a produção somada dos anos anteriores.

Para a categorização dos resultados, de acordo com o setor econômico, foi seguido o modelo de Barbieri et al. (2014), com a inclusão da classe $\mathrm{H}$ para agrupar as pesquisas realizadas junto a empresas de setores econômicos diversos, conforme a Tabela 3.

Ao analisar as particularidades do setor que se apresentou como mais profícuo, observou-se que a maioria os estudos (46,73\%) versou sobre aspectos de planejamento territorial e urbano, gestão logística $(20,10 \%)$, energia $(17,59 \%)$, telecomunicações $(13,07 \%)$ e construção civil $(2,51 \%)$.

Quanto ao objetivo, foi constatado que 123 trabalhos, aproximadamente 2/3 das 199 pesquisas que abordaram o setor econômico D - Infraestrutura, foram dedicados a seleção de variáveis, tais como modais de transporte, fornecedores de sistemas computacionais, projetos de traçado de rodovias e ferrovias, metodologias de configuração e distribuição de energia, ferramentas de gestão financeira, instrumentos de políticas públicas para a gestão de recursos hídricos, áreas para construção de aeroportos e destinação de resíduos, estratégias de exploração de recursos naturais, entre outros. 
Tabela 3 - Setores econômicos e temas abordados nas pesquisas

\begin{tabular}{|c|c|c|c|c|}
\hline \multirow[b]{2}{*}{ Setor econômico } & \multirow[b]{2}{*}{ Descrição } & \multicolumn{3}{|c|}{ Pesquisas empíricas } \\
\hline & & Tipo & Quantidade & $\begin{array}{l}\text { Fre- } \\
\text { quência } \\
(\%)\end{array}$ \\
\hline $\begin{array}{l}\text { A - Indústria de } \\
\text { base }\end{array}$ & $\begin{array}{l}\text { Cimenteiro, madeireiro, } \\
\text { de mineração, papel e } \\
\text { celulose, petróleo e gás, } \\
\text { plástico, químico, side- } \\
\text { rurgia e sucroalcooleiro. }\end{array}$ & $\begin{array}{l}\mathrm{D} \\
\mathrm{T}\end{array}$ & $\begin{array}{l}19 \\
1\end{array}$ & $\begin{array}{l}4,96 \\
0,26\end{array}$ \\
\hline $\begin{array}{l}\text { B - Bens de con- } \\
\text { sumo duráveis }\end{array}$ & $\begin{array}{l}\text { Automobilístico, ele- } \\
\text { troeletrônico, gráfico, } \\
\text { informática, moveleiro e } \\
\text { de manufatura. }\end{array}$ & $\mathrm{D}$ & 27 & 7,05 \\
\hline $\begin{array}{l}\mathrm{C}-\text { Bens de } \\
\text { consumo não } \\
\text { duráveis }\end{array}$ & $\begin{array}{l}\text { Setor agrícola, de ali- } \\
\text { mentos, bebidas, couro, } \\
\text { embalagens e farmacêu- } \\
\text { tico. }\end{array}$ & $\begin{array}{l}\mathrm{D} \\
\mathrm{T}\end{array}$ & $\begin{array}{l}30 \\
10\end{array}$ & $\begin{array}{l}7,83 \\
2,61\end{array}$ \\
\hline $\begin{array}{l}\text { D - Infraestru- } \\
\text { tura }\end{array}$ & $\begin{array}{l}\text { Planejamento territorial } \\
\text { e urbano, construção } \\
\text { civil, energia, telecomu- } \\
\text { nicações e transporte. }\end{array}$ & $\begin{array}{l}\mathrm{D} \\
\mathrm{T}\end{array}$ & $\begin{array}{l}159 \\
40\end{array}$ & $\begin{array}{l}41,51 \\
10,44\end{array}$ \\
\hline E - Serviços & $\begin{array}{l}\text { Ensino superior, hote- } \\
\text { laria, saúde e serviços } \\
\text { diversos governamen- } \\
\text { tais e privados. }\end{array}$ & $\begin{array}{l}\mathrm{D} \\
\mathrm{T}\end{array}$ & $\begin{array}{l}49 \\
11\end{array}$ & $\begin{array}{l}12,79 \\
2,87\end{array}$ \\
\hline F - Varejo & Comércio em geral. & $\begin{array}{l}\mathrm{D} \\
\mathrm{T}\end{array}$ & $\begin{array}{l}6 \\
2 \\
\end{array}$ & $\begin{array}{l}1,57 \\
0,52\end{array}$ \\
\hline $\begin{array}{l}\text { G - Metal-mecâ- } \\
\text { nico }\end{array}$ & $\begin{array}{l}\text { Transformação de me- } \\
\text { tais em produtos, má- } \\
\text { quinas e equipamentos. }\end{array}$ & $\begin{array}{l}\mathrm{D} \\
\mathrm{T}\end{array}$ & $\begin{array}{l}12 \\
1\end{array}$ & $\begin{array}{l}3,13 \\
0,26\end{array}$ \\
\hline $\begin{array}{l}\mathrm{H}-\text { Multisseto- } \\
\text { rial }\end{array}$ & $\begin{array}{l}\text { Refere-se a pesquisas } \\
\text { empíricas que foram } \\
\text { realizadas junto a em- } \\
\text { presas representantes de } \\
\text { duas ou mais categorias. }\end{array}$ & $\begin{array}{l}\mathrm{D} \\
\mathrm{T}\end{array}$ & $\begin{array}{l}10 \\
6\end{array}$ & $\begin{array}{l}2,61 \\
1,57\end{array}$ \\
\hline
\end{tabular}

Legenda: na coluna Tipo estão representadas dissertações (D) e teses (T).

Fonte: Elaborada pelos autores a partir dos dados coletados 
Verificou-se que os 41 estudos voltados à gestão de riscos se concentraram em aspectos de segurança na manipulação e armazenamento de dados em redes, mapeamento e modelagem espacial de áreas suscetíveis à movimentos de massa, escorregamentos, inundações e poluição do ar, vulnerabilidade ambiental ao impacto do transporte rodoviário e por meio de dutos.

Outros 35 trabalhos empregaram o método AHP para mensuração de indicadores operacionais e de qualidade, avaliação de desempenho de modais de transporte, matrizes energéticas e sistemas construtivos, análise do uso de tecnologias digitais na gestão de recursos naturais, áreas de preservação permanente e avaliação de impacto ambiental.

Na Figura 5 está representada a rede temática do método AHP no ambiente de pesquisas stricto sensu.

Figura 5 - Rede temática

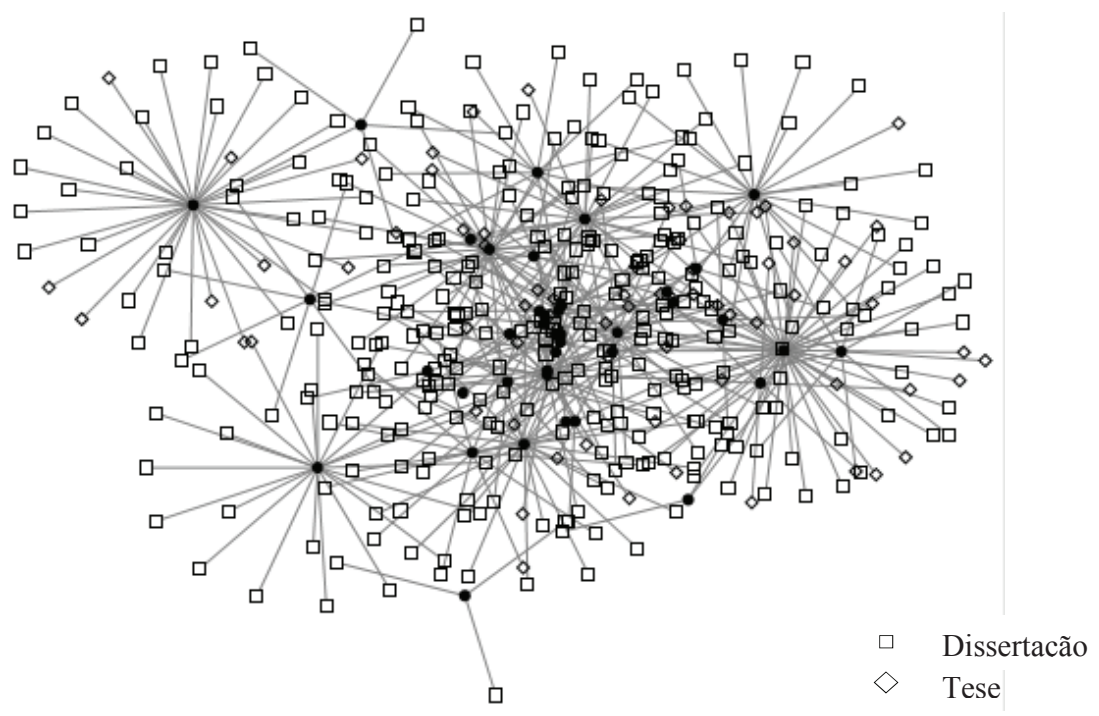

Fonte: Elaborada pelos autores a partir dos dados coletados

Em termos de proporção, foram verificadas como mais produtivas no emprego do método AHP em pesquisas acadêmicas as áreas 
de Engenharias e Ciências Sociais Aplicadas, que, juntas, somaram mais de $85 \%$ do total de trabalhos. Menor participação coube à área de Ciências da Saúde com 0,52\%. Além disso, não foram identificados estudos que representassem as áreas de Ciências Biológicas e Linguística, Letras e Artes.

Constatou-se sobre a produtividade das 40 instituições identificadas que a maioria (72,5\%) publicou menos de dez pesquisas no período. A mesma perspectiva de análise em relação aos 76 programas de pós-graduação retornou a taxa de 85,5\% deles abaixo de dez publicações. Ainda sob um olhar generalista a respeito de programas e instituições, observou-se respectivamente as frações $55 \%$ e $20 \%$ de ambos associados a uma única pesquisa.

Na Tabela 4, além da representação gráfica, são exibidas as métricas que permitiram a consolidação dos resultados mais expressivos.

A centralidade de grau indegree permite a contagem das relações direcionadas para o elemento. As áreas do conhecimento apresentam valor $=0$ porque delas parte o mapeamento e no caso dos programas de pós-graduação, como não foram considerados aspectos multidisciplinares, o valor 1 remete à área do conhecimento a que pertencem. O grau indegree proporciona uma análise mais aprofundada do desempenho das instituições no tocante a disseminação do método AHP entre os seus programas.

Por meio do valor da centralidade de grau outdegree verificam-se as ligações estabelecidas a partir do elemento. A área de Engenharias foi identificada como mais proficiente, pois reuniu o maior número de programas com desenvolvimento de pesquisas sobre AHP. A mesma observação é feita em relação ao programa de pós-graduação em Engenharia de Produção que apresentou o maior número de pesquisas distribuídas entre 14 instituições. A avaliação do grau de centralidade outdegree para as instituições de ensino faculta o reconhecimento daquelas que possuem os valores mais altos como as mais profícuas.

Os demais parâmetros apresentados se prestam à confirmação e interpretação mais aprofundadas dos resultados. A participação de um elemento em uma rede pode ser quantificada por meio da interpretação do grau de intermediação que corresponde à contagem 
do número de vezes que esse elemento atuou como ponte entre os demais (FREEMAN, 1996).

A influência de um nó é medida pelos valores da centralidade do autovetor que atribuem pontuação ao nó relativa a todos os demais nós da rede, maior se as ligações são estabelecidas com outros nós de pontuação alta e, menores quando as ligações são firmadas com nós de pontuação mais baixa (BONACICH, 2007).

Por fim, tem-se a classificação de importância de um elemento por meio do cálculo da qualidade e quantidade de informação sobre ele, obtida por meio dos outros nós (PAGE et al., 1999). Os principais resultados são apresentados na Tabela 4.

Tabela 4 - Principais resultados da aplicação das métricas de ARS

\begin{tabular}{|c|c|c|c|c|c|c|}
\hline \multicolumn{7}{|c|}{ Áreas do conhecimento } \\
\hline \multirow{2}{*}{ Descrição } & \multirow{2}{*}{ Subgrafo } & \multicolumn{2}{|c|}{$\begin{array}{l}\text { Grau de centrali- } \\
\text { dade }\end{array}$} & \multirow{2}{*}{$\begin{array}{l}\text { Grau de in- } \\
\text { termediação }\end{array}$} & \multirow{2}{*}{$\begin{array}{l}\text { Centrali- } \\
\text { dade do } \\
\text { autovetor }\end{array}$} & \multirow{2}{*}{$\begin{array}{l}\text { Classificação de } \\
\text { importância } \\
\text { Pagerank }\end{array}$} \\
\hline & & Indegree & Outdegree & & & \\
\hline Engenharias & & 0 & 30 & $49.548,29$ & 0,013 & 9.820 \\
\hline $\begin{array}{l}\text { Ciências } \\
\text { Sociais e Apli- } \\
\text { cadas }\end{array}$ & & 0 & 24 & $24.281,84$ & 0,008 & 7.806 \\
\hline \multicolumn{7}{|c|}{ Programas de pós-graduação } \\
\hline Descrição & Subgrafo & \multicolumn{2}{|c|}{$\begin{array}{l}\text { Grau de centrali- } \\
\text { dade }\end{array}$} & $\begin{array}{l}\text { Grau de in- } \\
\text { termediação }\end{array}$ & $\begin{array}{l}\text { Centrali- } \\
\text { dade do } \\
\text { autovetor }\end{array}$ & Classificação \\
\hline $\begin{array}{l}\text { Engenharia de } \\
\text { Produção }\end{array}$ & & 1 & 14 & $67.217,97$ & 0,017 & 5.023 \\
\hline $\begin{array}{l}\text { Engenharia } \\
\text { Civil }\end{array}$ & & 1 & 10 & $36.206,09$ & 0,013 & 3.877 \\
\hline Administração & & 1 & 8 & $21.365,064$ & 0,012 & 3.123 \\
\hline
\end{tabular}




\begin{tabular}{|c|c|c|c|c|c|c|}
\hline $\begin{array}{l}\text { Engenharia } \\
\text { Elétrica }\end{array}$ & & 1 & 6 & $16.162,75$ & 0,007 & 2.383 \\
\hline $\begin{array}{l}\text { Gestão Am- } \\
\text { biental }\end{array}$ & & 1 & 11 & $15.874,22$ & 0,011 & 4.054 \\
\hline $\begin{array}{l}\text { Gestão de } \\
\text { Projetos }\end{array}$ & & 1 & 8 & $12.168,46$ & 0,012 & 2.922 \\
\hline \multicolumn{7}{|c|}{ Instituições de ensino superior } \\
\hline Descrição & Subgrafo & \multicolumn{2}{|c|}{$\begin{array}{l}\text { Grau de centrali- } \\
\text { dade }\end{array}$} & $\begin{array}{l}\text { Grau de } \\
\text { intermedia- } \\
\text { ção }\end{array}$ & $\begin{array}{l}\text { Centrali- } \\
\text { dade do } \\
\text { autovetor }\end{array}$ & Classificação \\
\hline USP & & 20 & 51 & $64.240,54$ & 0,039 & 26.551 \\
\hline UNESP & & 8 & 34 & $37.842,53$ & 0,012 & 16.608 \\
\hline PUC & & 8 & 26 & 35831,11 & 0,009 & 13.026 \\
\hline UNB & & 11 & 29 & $31.983,69$ & 0,002 & 15.161 \\
\hline UFSC & & 6 & 28 & $29.736,09$ & 0,007 & 13.618 \\
\hline UNICAMP & & 7 & 24 & $26.734,16$ & 0,005 & 12.170 \\
\hline UFRGS & & 3 & 22 & $26.489,41$ & 0,005 & 10.631 \\
\hline
\end{tabular}




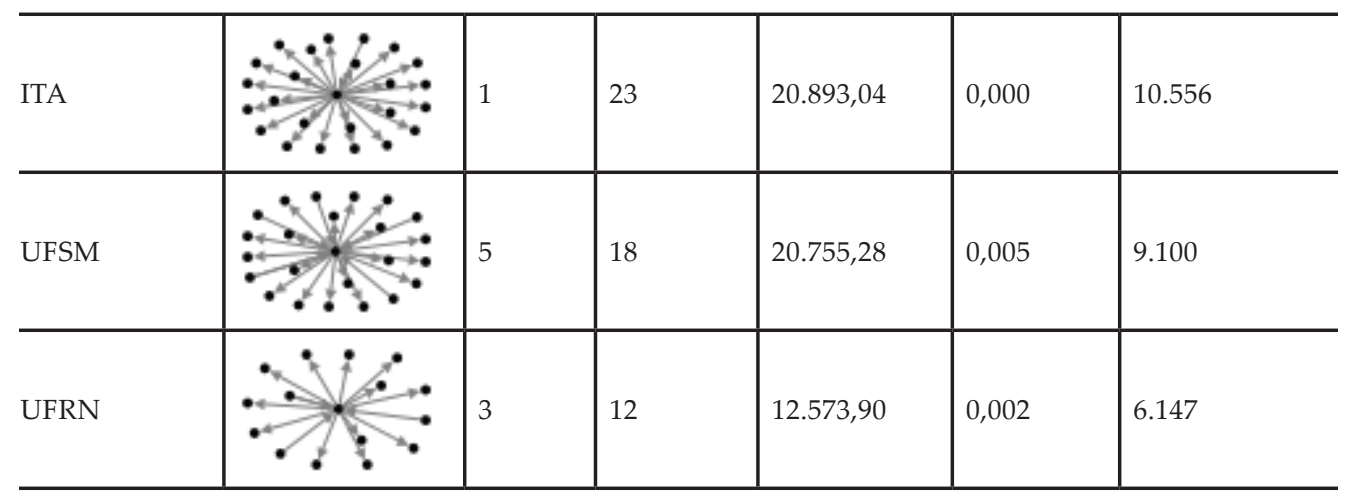

Fonte: Elaborada pelos autores a partir dos dados coletados

No que se refere a aderência dos professores ao método, verificou-se que os casos únicos de orientação foram predominantes e alcançaram 223 dentre o total de 383 pesquisas. Entre as dez instituições mais prolíficas, que, juntas, respondem por, aproximadamente, $70 \%$ dos estudos desenvolvidos, 155 trabalhos corresponderam aos casos únicos de orientação. Dezesseis professores orientaram dois estudos, doze responderam pela orientação de três pesquisas, e três professores orientaram quatro trabalhos.

Os pesquisadores mais proficientes, com orientação de 5, 7 e 11 estudos envolvendo o uso do método AHP, foram identificados nas instituições (Universidade Federal do Rio Grande do Norte (UFRN), Pontifícia Universidade Católica (PUC) e Instituto Tecnológico de Aeronáutica (ITA), respectivamente.

Em relação ao tipo de abordagem, observou-se a prevalência do uso do método AHP em sua forma clássica, tal como descrito nas etapas 1 a 11 do item 2.2, em mais de 2/3 dos estudos. A aplicação conjunta com métodos diversos MAUTH, MACBEHT, TOPSIS, TODIM, SMART, ELECTRE, PROMETHEE, entre outros, somou, aproximadamente, $14 \%$ dos trabalhos. A associação do AHP com a lógica fuzzy, o F-AHP, foi verificada em cerca de 3,5\% dos trabalhos, e as conjunções com procedimentos, tais como programação matemática linear, desdobramento da função qualidade ou Quality Function Deployment (QFD) e análise envoltória de dados Data Envelopment Analysis (DEA), foram identificadas em menos de $2 \%$ das pesquisas. 
A respeito das ferramentas utilizadas, constatou-se que a maioria dos pesquisadores empregaram planilhas eletrônicas na elaboração das matrizes de cálculo, cerca de 70\% dos mestrandos e mais de $85 \%$ dos doutorandos. Dentre as ferramentas sistematizadas utilizadas, cerca de $65 \%$ da preferência permaneceu com o software Expert Choice. Também foram identificadas em seis pesquisas o desenvolvimento e a aplicação de ferramentas próprias para o uso do método AHP.

\section{DisCuSSÃo doS RESUlTAdos}

A proeminência de aplicação da versão tradicional reafirma o estágio embrionário do AHP como metodologia de pesquisa. Segundo Vaidya e Kumar (2006), passados os estágios iniciais e à medida que os pesquisadores evoluem no domínio da técnica, as combinações do AHP com outros métodos tornam-se mais frequentes.

Os arranjos metodológicos observados entre os resultados foram adotados pela maioria dos pesquisadores com o objetivo de comparar e confirmar os resultados obtidos. Há mais de uma década, as integrações com o método AHP mais comumente encontradas nas publicações de periódicos internacionais incluíram ferramentas meta-heurísticas, tais como algoritmos genéticos e redes neurais artificiais, análise SWOT e DEA, e principalmente função QFD e programação matemática $(\mathrm{HO}, 2008)$. Entre os achados, tais tipos de conjunções metodológicas foram identificadas em menos de dez pesquisas.

Em recente atualização sobre as integrações com o AHP mais utilizadas, Ho e Ma (2018) identificaram o F-AHP como o mais comum no período de 2007-2016, bem como a tendência de combinar mais de uma técnica com o método AHP.

Em relação às possibilidades de exploração das diversas áreas do conhecimento por meio do método AHP, confronta-se em especial os resultados daquelas que obtiveram menor representatividade com exemplos de pesquisas publicadas na literatura mundial.

Em saúde, o estudo de Liberatore e Nydick (2008) identificou, no período 1988-2007, 50 artigos relacionados a tomada de decisão em procedimentos médicos e cuidados com a saúde, tais como diagnose, terapias e tratamento, transplante de órgãos, seleção de projetos e tecnologias, planejamento de recursos humanos e avalia- 
ção de políticas de cuidados na saúde. Esses autores distinguiram o AHP como uma importante ferramenta de integração entre médico e paciente na determinação estratégias de tratamento e, além disso, constataram uma taxa de aumento de três artigos por ano nos últimos dez anos do período estudado.

Uma vez que não foram identificadas pesquisas da área de ciências biológicas relacionadas ao método AHP, entre outros, sugerem-se os exemplos de utilização: a) AHP clássico na avaliação da adequação de habitat para aves aquáticas (DONG et al., 2013), análise de risco da disseminação de gripe aviária altamente patogênica (KOU et al., 2009) e gestão de árvores invasoras (ROURA-PASCUAL et al., 2009); e b) F-AHP na avaliação dos benefícios da comercialização de recursos genéticos vegetais (LEE; SOHN, 2016), e do potencial energético da digestão anaeróbica de resíduos de culturas (ESCALANTE et al., 2016).

As designações da escala de julgamentos oferecem à área de Linguística subsídios suficientes para o estudo do método AHP, bem como as áreas de Letras e Artes podem se utilizar dos diversos exemplos anteriormente relatados.

\section{CONSIDERAÇões FINAIS}

Foi constatado que os anos de 2012 a 2016 respondem por cerca de 55\% da utilização do método AHP em pesquisas de pós-graduação stricto sensu. Visto que o AHP se utiliza de um modo intuitivo de resolução de problemas e possui aplicação facilitada pela possibilidade de modelagem do problema e utilização de julgamento verbais (ISHIZAKA; LABIB, 2011), e, a considerar pelo histórico de evolução da pós-graduação stricto no que se refere ao aumento do número de programas oferecidos (CIRANI; CAMPANÁRIO; SILVA, 2015) tanto acadêmicos quanto profissionais, estima-se que o AHP seja mais difundido nos anos futuros.

O mapeamento constatou também, a predominância da aplicação do método em sua versão clássica, e considerada a identificação de poucos nichos produtivos, infere-se que as incursões ao método AHP apresentam, em sua maioria, caráter isolado e experimental.

Visto que ainda são poucas as publicações em português, este estudo contribui para a teoria ao apresentar aos interessados no AHP 
sua fundamentação teórica e axiomática, além disso, serve como um sumário que facilite aos pesquisadores 383 fontes de consulta e novos insights de pesquisa.

A respeito das contribuições trazidas por este estudo, ao detalhar a aplicação do AHP em onze etapas, este trabalho faculta a utilização do método em termos práticos, não somente para fins acadêmicos como para as várias situações em que seja necessária a tomada de decisão por multicritérios.

\section{REFERÊNCIAS}

ABASTANTE, F; CORRENTE, S; GRECO, S; IZHIZAKA, A; LAMI, R. A new parsimonious AHP methodology: Assigning priorities to many objects by comparing pairwise few reference objects. Expert Systems With Applications, v. 127, p. 109-20, 2019. https://doi. org/10.1016/j.eswa.2019.02.036.

BALUSA, B. C.; GORAI, A. K. Sensitivity analysis of fuzzy-analytic hierarchical process (FAHP) decision-making model in selection of underground metal mining method. Journal of Sustainable Mining, v. 18, n. 1, p. 8-17, 2019. https://doi.org/10.1016/j.jsm.2018.10.003.

BANA e COSTA, C. A.; VANSNICK, J. C. A critical analysis of the eigenvalue method used to derive priorities in AHP. European Journal of Operational Research, v. 187, n. 3, p. 1422-28, 2008. https://doi.org/10.1016/j.ejor.2006.09.022.

BARBIERI, J. C; SOUSA FILHO, J. M; BRANDÃO, C.N; DI SÉRIO, L. C.; REYES JUNIOR, E. Gestão verde da cadeia de suprimentos: análise da produção acadêmica brasileira. Revista Produção Online, v. 14, n. 3, p. 1104-28, 2014. https://doi.org/10.14488/16761901.v14i3.16.

BARIN, A; CANHA, L. N; MAGNAGO, K. F.; ABAIDE, A. R. Seleção de fontes alternativas de geração distribuída utilizando uma análise multicriterial baseada no método AHP e na lógica fuzzy. Controle \& Automação, v. 21, n. 5, p. 477-86, 2010. http://dx.doi.org/10.1590/ S0103-17592010000500004.

BARZILAI, J.; GOLANY, B. AHP rank reversal, normalization and aggregation rules. INFOR - Information Systems and Operation Research, v. 32, n. 2, p. 57-64, 1994. https:// doi.org/10.1080/03155986.1994.11732238.

BELTON, V. A.; GEAR, T. The legitimacy of rank reversal. Omega, v. 13, n. 3, p. 143-44, 1985. https://doi.org/10.1016/0305-0483(85)90052-0.

BELTON, V.; GOODWIN, P. Remarks on the application of the analytic hierarchy process to judgmental forecasting. International Journal of Forecasting, v. 12, n. 1, p. 155-61, 1996. https://doi.org/10.1016/0169-2070(95)00643-5.

BELTON, V.; STEWART, T. Multiple criteria decision analysis: an integrated approach. Norwell: Kluwer Academic Publishers, 2002. 
BENÍTEZ, J. CARPITELLA, S; CERTA, A; IZQUIERDO, J. Management of uncertain pairwise comparisons in AHP through probabilistic concepts. Applied Soft Computing Journal, v. 78, p. 274-85, 2019. https://doi.org/10.1016/j.asoc.2019.02.020.

BONACICH, P. Some unique properties of eigenvector centrality. Social networks, v. 29, n. 4, p. 555-64, 2007. https://doi.org/10.1016/j.socnet.2007.04.002.

BOUYSSOU, D. Building criteria: a prerequisite for MCDA. In: Bana e Costa, C. A. Readings in Multiple Criteria Decision Aid. Berlin: Springer-Verlag, 1990, 58-79.

BUCKLEY, J. J. Fuzzy hierarchical analysis. Fuzzy Sets and Systems, v. 17, n. 3, p. 233-47, 1985. https://doi.org/10.1016/0165-0114(85)90090-9.

CHANG, D. Y. Applications of the extent analysis method on fuzzy AHP. European Journal of Operational Research, v. 95, n. 3, p. 649-55, 1996. https://doi.org/10.1016/03772217(95)00300-2.

CIRANI, C.; CAMPANARIO, M.; SILVA, H. H. A evolução do ensino da pós-graduação senso estrito no Brasil: análise exploratória e proposições para pesquisa. Avaliação: Revista da Avaliação da Educação Superior, v. 20, n. 1, p. 163-87, 2015. http://dx.doi.org/10.590/ S1414-40772015000500011.

DENG, H. Multicriteria analysis with fuzzy pairwise comparison. International Journal of Approximate Reasoning, v. 21, n. 3, p. 215-31, 1999. https://doi.org/10.1016/S0888-613X(99)00025-0.

DONG, Z. WANG, Z.; LIU, D; LI, L; REN, C; TANG, X; JA, M.; LIU, C. Assessment of habitat suitability for waterbirds in the West Songnen Plain, China, using remote sensing and GIS. Ecological Engineering, v. 55, p. 94-100, 2013. https://doi.org/10.1016/j.ecoleng.2013.02.006.

ESCALANTE, H; CASTRO, L GAUTHIER-MARADEI, P; DE LA VEGA, R. R. Spatial decision support system to evaluate crop residue energy potential by anaerobic digestion. Bioresource Technology, v. 219, p. 80-90, 2016. https://doi.org/10.1016/j.biortech.2016.06.136.

FREEMAN, L. C. Some antecedents of Social Networks Analysis. Connections, v. 19, n. 1, p. 39-42, 1996.

FUNO, K. A.; MUNIZ JR., J.; MARINS, F. A. S. Fatores de risco em cadeia de suprimentos do setor aeroespacial: aspectos qualitativos e quantitativos. Produção, v. 23, n. 4, p. 832-45, 2013. http://dx.doi.org/10.1590/S0103-65132013005000016.

GOMES, L. F. A. M.; GOMES, C. F. S.; ALMEIDA, A. T. Tomada de decisão gerencial: enfoque multicritério. 3. ed. São Paulo: Atlas, 2009.

HANSEN, D.; SHNEIDERMAN, B.; SMITH, M. Analyzing social media networks with NodeXL. San Francisco: Elsevier Science \& Technology, 2010.

$\mathrm{HO}, \mathrm{W}$. Integrated analytic hierarchy process and its applications - A literature review. European Journal of Operational Research, v. 186, n. 1, p. 211-28, 2008. https://doi.org/10.1016/j. ejor.2007.01.004.

HO, W.; MA, X. The state-of-the-art integrations and applications of the analytic hierarchy process. European- Journal of Operational Research, v. 267, n. 2, p. 399-414, 2018. https:// doi.org/10.1016/j.ejor.2017.09.007. 
ISHIZAKA, A.; LABIB, A. Analytic hierarchy process and expert choice: Benefits and limitations. OR Insight, v. 22, n. 4, p. 201-20, 2009. https://doi.org/10.1057/ori.2009.10.

ISHIZAKA, A.; LABIB, A. Review of the main developments in the Analytic Hierarchy Process. Expert Systems with Applications, v. 38, n. 11, p. 14336-45, 2011. https://doi. org/10.1016/j.eswa.2011.04.143.

KHATWANI, G.; KAR, A. K. Improving the Cosine Consistency Index for the analytic hierarchy process for solving multi-criteria decision making problems. Applied Computing and Informatics, v. 13, n. 2, p. 118-29, 2017. https://doi.org/10.1016/j.aci.2016.05.001.

KOU, Z; LI, Y; YIN, Z; GUO, S; WANG, M; GAO, X; LI,P; TANG, L; JIANG, P; LUO, Z; XIN, Z; DING; C; HE, Y; REN, Z; CUI, P; ZHAO, H; ZHANG, Z; TANG, S; YAN, $\mathrm{B}$; LEI, F; LI, T. The survey of H5N1 flu virus in wild birds in 14 Provinces of China from 2004 to 2007. PLoS One, v. 4, n. 9, p. 1-8, 2009. https://doi.org/10.1371/journal.pone.0006926.

LEE, B. K.; SOHN, S. Y. Patent portfolio-based indicators to evaluate the commercial benefits of national plant genetic resources. Ecological Indicators, v. 70, p. 43-52, 2016. https:// doi.org/10.1016/j.ecolind.2016.05.002.

LIBERATORE, M. J.; NYDICK, R. L. The analytic hierarchy process in medical and health care decision making: A literature review. European Journal of Operational Research, v. 189, n. 1, p. 194-207, 2008. https://doi.org/10.1016/j.ejor.2007.05.001.

LIEGGIO JR. M.; GRANEMANN, S. R.; SOUZA, O. A. Aplicabilidades da análise multicritério às problemáticas de decisão no transporte rodoviário de produtos perigosos: uma perspectiva teórica. Journal of Transport Literature, v. 6, n. 2, p. 197-217, 2012. http:// dx.doi.org/10.1590/S2238-10312012000200011.

LIMA, J. D; JUCÁ, J. F. T; REICHERT, G. A; FIRMO, A. L. B. Uso de modelos de apoio à decisão para análise de alternativas tecnológicas de tratamento de resíduos sólidos urbanos na Região Sul do Brasil. Engenharia Sanitária e Ambiental, v. 19, n. 1, p. 33-42, 2014. http:// dx.doi.org/10.1590/S1413-41522014000100004.

LOOTSMA, F. A. The French and the American school in multi-criteria decision analysis. Recherche opérationnelle/Operations Research, v. 24, n. 3, p. 263-85, 1990.

MATOS, P. V; CARDADEIRO, E; SILVA, J. A; MUYLDER, C. F. The use of multi-criteria analysis in the recovery of abandoned mines: a study of intervention in Portugal. RAUSP Management Journal, v. 53, n. 2, p. 214-24, 2018. http://dx.doi.org/10.1016/j.rauspm.2017.06.005.

MILLET, I.; SAATY, T. L. On the relativity of relative measures - accommodating both rank preservation and rank reversals in the AHP. European Journal of Operational Research, v. 121, n. 1, p. 205-12, 2000. https://doi.org/10.1016/S0377-2217(99)00040-5.

NAGHADEHI, M. Z.; MIKAEIL, R.; ATAEI, M. The application of fuzzy analytic hierarchy process (FAHP) approach to selection of optimum underground mining method for Jajarm Bauxite Mine, Iran. Expert Systems with Applications, v. 36, n. 4, p. 8218-26, 2009. https://doi.org/10.1016/j.eswa.2008.10.006.

OSSADNIK, W.; LANGE, O. AHP-based evaluation of AHP-Software. European Journal of Operational Research, v. 118, n. 3, p. 578-88, 1999. https://doi.org/10.1016/S0377$-2217(98) 00321-X$. 
PAGE, L; BRIN, S; MONWANI, R; WINOGRAD, T. The PageRank citation ranking: Bringing order to the web. University of Southampton, England: Stanford InfoLab, 1999.

PARREIRAS, R. O. (2006). Algoritmos evolucionários e técnicas de tomada de decisão em análise multicritério. Belo Horizonte, 2006. 166f. Tese (Doutorado) - Escola de Engenharia da UFMG, Universidade Federal de Minas Gerais.

PRODANOV, C. C.; FREITAS, E. C. Metodologia do trabalho científico: métodos e técnicas da pesquisa e do trabalho acadêmico. 2. ed. Novo Hamburgo: Universidade FEEVALE, 2013.

ROURA-PASCUAL, N; RICHARDSON, D. M; KRUG, R M; BROWN, A; CHAPMAN, R. A; FORSYTH, G. C; LE MAITRE, D. C; ROBERTSON, M, P; STAFFORD, L; VAN WILGEN, B. W; WANENBURGH, A; WESSELS, N. Ecology and management of alien plant invasions in South African fynbos: accommodating key complexities in objective decision making. Biological Conservation, v. 142, n. 8, p. 1595-604, 2009. https://doi.org/10.1016/j. biocon.2009.02.029.

SAATY, T. L. A scaling method for priorities in hierarchical structures. Journal of Mathematical Psychology, v. 15, n. 3, p. 234-81, 1977. https://doi.org/10.1016/0022-2496(77)90033-5.

SAATY, T. L. Axiomatic foundation of the analytic hierarchy process. Management Science, v. 32, p. 7, p. 841-55, 1986.

SAATY, T. L. The analytic hierarchy process - what it is and how it is used. Mathematical Modelling, v. 9, n. 3-5, p. 161-76, 1987. https://doi.org/10.1016/0270-0255(87)90473-8.

SAATY, T. L. How to make a decision: the Analytic Hierarchy Process. European Journal of Operational Research, v. 48, n. 1, p. 9-26, 1990. https://doi.org/10.1016/0377-2217(90)90057-I.

SAATY, T. L. Response to Holder's comments on the Analytic Hierarchy Process. The Journal of the Operational Research Society, v. 42, n. 10, p. 909-14, 1991. https://doi.org/10.1057/ jors.1991.176.

SAATY, T. L. Highlights and critical points in the theory and application of the analytic hierarchy process. European Journal of Operational Research, v. 74, n. 3, p. 426-47, 1994. https://doi.org/10.1016/0377-2217(94)90222-4.

SAATY, T. L. Rank from comparisons and from ratings in the analytic hierarchy/network processes. European Journal of Operational Research, v. 168, n. 2, p. 557-70, 2006. https:// doi.org/10.1016/j.ejor.2004.04.032.

SAATY, T. L. Analytical planning: the organization of systems. In: Kearns, K. P. International Series in Modern Applied Mathematics and Computer. 7. ed. New York: Pergamon Press, 2014.

SAATY, T. L.; HU, G. Ranking by eigenvector versus other methods in the Analytic Hierarchy Process. Applied Mathematics Letters, v. 11, n. 4, p. 121-25, 1998. https://doi.org/10.1016/ S0893-9659(98)00068-8.

SAATY, T. L.; VARGAS, L. G. Models, methods, concepts e applications of the Hierarchy Analysis Process. 2. ed. New York: Springer Science \& Business Media, 2012a.

SAATY, T. L.; VARGAS, L. G. The seven pillars of the analytic hierarchy process. In: Models, methods, concepts \& applications: applications of the Analytic Hierarchy Process. New York: Springer Science \& Business Media, 2012b, v. 175, 23-40. 
SALOMON, V. A. P. (2010). Contribuições para tomada de decisão com múltiplos critérios. Guaratinguetá, 2010. 65f. Tese (Livre-Docência) - Faculdade de Engenharia, Universidade Estadual Paulista Júlio de Mesquita Filho.

SALOMON, V. A. P.; MONTEVECHI, J. A. B. A compilation of comparisons on the analytic hierarchy process and others multiple criteria decision making methods: some cases developed in Brazil. Proceedings of International Symposium on the Analytic Hierarchy Process, Bern, Switzerland, $6^{\text {th }}, 2001$.

SANTOS, P. H; NEVES, S. M; SANT'ANNA, D. O; OLIVEIRA, C. H; CARVALHO, H. D. The Analytic Hierarchy Process supporting decision making for sustainable development: an overview of applications. Journal of Cleaner Production, v. 212, p. 119-38, 2019. https:// doi.org/10.1016/j.jclepro.2018.11.270.

SOLTANI, A.; MARANDI, E. Z.; IVAKI, Y. E. Bus route evaluation using a two-stage hybrid model of Fuzzy AHP and TOPSIS. Journal of Transport Literature, v. 7, n. 3, p. 34-58, 2013. http://dx.doi.org/10.1590/S2238-10312013000300003.

STEIGUER, J. E.; DUBERSTEIN, J.; LOPES, V. The analytic hierarchy process as a means for integrated watershed management. In: Kenneth G Renard, First Interagency Conference on Research on the Watersheds, p. 736-40, 2003.

TREVISANO, W. A. Ferramenta computacional multiusuário para auxílio à tomada de decisão multicritério. Campos de Goytacazes, 2007. 110f. Dissertação (Mestrado) - Centro de Ciência e Tecnologia, Universidade Estadual do Norte Fluminense.

UNIKASARI, F; IFTADI, I; HAUHARI, JAUHARI, WA; DANARDONO, D. Study of the factors that affecting automobile seat comfort. Proceedings of Joint International Conference on Rural Information \& Communication Technology and Electric-Vehicle Technology. Bandung-Bali, Indonesia, 2013.

Van LAARHOVEN, P. J. M.; PEDRYCZ, W. A fuzzy extension of Saaty's priority theory. Fuzzy Sets and Systems, v. 11, n. 1-3, p. 229-41. 1983. https://doi.org/10.1016/S01650114(83)80082-7.

VARGAS, L. G. An overview of the Analytic Hierarchy Process and its applications. European Journal of Operational Research, v. 48. n. 1, p. 2-8, 1990. https://doi.org/10.1016/0377-2217(90)90056-H.

VAIDYA, O. S.; KUMAR, S. Analytic Hierarchy Process: An overview of applications. European Journal of Operational Research, v. 169, n. 1, p. 1-29, 2006. https://doi.org/10.1016/j. ejor.2004.04.028.

VIEIRA, A. M. Cultura organizacional em instituições de ensino: mapeamento e análise descritivo-interpretativa da produção acadêmica (1990-2005). 2007. 237f. Tese (Doutorado em Educação) - Universidade Estadual Paulista (UNESP), Marília, 2007.

WASSERMANN, S.; FAUST, K. Social network analysis: Methods and applications. Cambridge: Cambridge university press, 1994.

WATSON, S. R.; FREELING, A. N. S. Assessing attribute weights by ratios. Omega, v. 10, n. 6, p. 582-83, 1982. https://doi.org/10.1016/0305-0483(82)90061-5. 
WHITAKER, R. Criticisms of the Analytic Hierarchy Process: Why they often make no sense. Mathematical and Computer Modelling, v. 46, n. 7-8, p. 948-61, 2007. https://doi. org/10.1016/j.mcm.2007.03.016.

ZYOUD, S. H.; FUCHS-HANUSCH, D. A bibliometric-based survey on AHP and TOPSIS techniques. Expert Systems With Applications, v. 78, p. 158-81, 2017. https://doi.org/10.1016/j. eswa.2017.02.016.

Recebido em: 8-6-2019

Aprovado em: 12-12-2020

Avaliado pelo sistema double blind review.

Disponível em http://mjs.metodista.br/index.php/roc 